\title{
Sevoflurane preconditioning attenuates hypoxia/reoxygenation injury of H9c2 cardiomyocytes by activation of the HIF-1/PDK-1 pathway
}

\author{
Tianliang Hou ${ }^{\text {Equal first author, } 1}$, Haiping Ma ${ }^{\text {Equal first author, } 1}{ }^{\text {, Haixia Wang }}{ }^{2}$, Chunling Chen ${ }^{1}$, Jianrong Ye ${ }^{\text {Corresp., } 1}$, Ahmed \\ Mohamed Ahmed ${ }^{3}$, Hong Zheng ${ }^{\text {Corresp. } 1}$ \\ ${ }^{1}$ Department of anesthesiology, The First Affiliated Hospital of Xinjiang Medical University, Urumqi, Xinjiang, China \\ 2 Department of Mastology, Xinjiang Maternal and Child Health Hospital, Urumqi, Xinjiang, China \\ 3 Department of Intensive Care Unit (ICU), Yardimeli Hospital, Mogadishu, Somalia, Somalia \\ Corresponding Authors: Jianrong Ye, Hong Zheng \\ Email address: 616227972@qq.com, xj_zhenghong@sina.com
}

Background: Sevoflurane preconditioning (SPC) can provide myocardial protective effects similar to ischemic preconditioning(IPC). However, the underlying molecular mechanism of SPC remains unclear. Studies confirm that hypoxia-inducible factor-1 (HIF-1) can transform cells from aerobic oxidation to anaerobic glycolysis by activating the switch protein pyruvate dehydrogenase kinase-1 (PDK-1), thus providing energy for the normal life activities of cells under hypoxic conditions. The purpose of this study was to investigate whether the cardioprotective effects of SPC are associated with activation of the HIF-1a/PDK-1 signal pathway. Methods: The H9c2 cardiomyocytes hypoxia/reoxygenation model was established and treated with $2.4 \%$ sevoflurane at the end of equilibration. Lactate dehydrogenase (LDH) level, cell viability, cell apoptosis, mitochondrial membrane potential, key enzymes of glycolysis, ATP concentration of glycolysis were assessed after the intervention. Apoptosis related protein(Bcl-2, Bax), HIF-1a protein, and PDK-1 protein were assessed by western blot.Results: Compared with the H/R group, SPC significantly increased the expression of HIF-1a, PDK-1, and $\mathrm{BCl}-2$ and reduced the protein expression of Bax, which markedly decreased the apoptosis ratio and Lactate dehydrogenase (LDH) level, increasing the cell viability, content of key enzymes of glycolysis, ATP concentration of glycolysis and stabilizing the mitochondrial membrane potential. However, the cardioprotective effects of SPC were disappeared by treatment with a HIF-la selective inhibitor.Conclusion: This study demonstrates that the cardioprotective effects of SPC are associated with the activation of the HIF-1a/PDK-1 signaling pathway. The mechanism may be related to increasing the content of key enzymes and ATP of glycolysis in the early stage of hypoxia.Keywords: Sevoflurane preconditioning, hypoxia/reoxygenation injury, myocardial protective effect, hypoxia-inducible factor-1, pyruvate dehydrogenase 
kinase-1, glycolysis 


\section{Sevoflurane preconditioning attenuates hypoxia/reoxygenation injury of H9c2}

\section{2 cardiomyocytes by activation of the HIF-1/PDK-1 pathway}

3 Tianliang Hou $^{\mathrm{a},{ }^{*}}$, Haiping Ma $\mathrm{a}^{\mathrm{a},}$, Haixia Wang ${ }^{\mathrm{b}}$, Chunling Chen ${ }^{\mathrm{a}}$, Jianrong Ye ecorresp., Ahmed Mohamed Ahmed,

4 Hong Zheng,

5 a Department of Anesthesiology, The Frist Affiliated Hospital of Xinjiang Medical University, Urumqi, Xinjiang,

6 China;

$7 \quad{ }^{b}$ Department of Mastology, Xinjiang Maternal, and Child Health Hospital, Urumqi, Xinjiang, China;

$8 \quad{ }^{\mathrm{c}}$ Department of Intensive Care Unit (ICU), Yardimeli Hospital, Mogadishu, Somalia

10 Corresponding authors: Prof. Hong Zheng and Jianrong Ye, Department of Anesthesiology, The First Affiliated 11 Hospital of Xinjiang Medical University, No. 137, Liyushan South Street, Urumqi, Xinjiang, 830054, China, 12 Tel: +86-0991-4366167, E-mail: xjzhenghong@sina.com, E-mail: $\underline{616227972 @ \text { qq.com }}$

\section{Abstract}

Background: Sevoflurane preconditioning (SPC) can provide myocardial protective effects similar to ischemic preconditioning (IPC). However, the underlying molecular mechanism of SPC remains unclear. Studies confirm that hypoxia-inducible factor-1 (HIF-1) can transform cells from aerobic oxidation to anaerobic glycolysis by activating the switch protein pyruvate dehydrogenase kinase-1 (PDK-1), thus providing energy for the normal life activities of cells under hypoxic conditions. The purpose of this study was to investigate whether the cardioprotective effects of SPC are associated with activation of the HIF-1a/PDK-1 signal pathway.

Methods: The H9c2 cardiomyocytes hypoxia/reoxygenation model was established and treated with $2.4 \%$ sevoflurane at the end of equilibration. Lactate dehydrogenase (LDH) level, cell viability, cell apoptosis, mitochondrial membrane potential, key enzymes of glycolysis, ATP concentration of glycolysis were assessed after the intervention. Apoptosis related protein (Bcl-2, Bax), HIF1a protein, and PDK-1 protein were assessed by western blot.

Results: Compared with the H/R group, SPC significantly increased the expression of HIF-1a, PDK-1, and Bcl-2 and reduced the protein expression of Bax, which markedly decreased the apoptosis ratio and Lactate dehydrogenase (LDH) level, increasing the cell viability, content of key enzymes of glycolysis, ATP concentration of glycolysis and stabilizing the mitochondrial membrane 
28 Conclusion: This study demonstrates that the cardioprotective effects of SPC are associated with the activation of the HIF-

1a/PDK-1 signaling pathway. The mechanism may be related to increasing the content of key enzymes and ATP of glycolysis in the early stage of hypoxia.

Keywords: Sevoflurane preconditioning, hypoxia/reoxygenation injury, myocardial protective effect, hypoxia-inducible factor1, pyruvate dehydrogenase kinase-1, glycolysis

4 Introduction

Despite substantial progress in prevention and treatment, ischemic heart disease (IHD) remains the main cause of morbidity and mortality worldwide (Devereaux et al. 2017). At present reperfusion therapy is the most effective treatment for IHD. However, it is also inevitable that ischemia/reperfusion (I/R) injury will occur to cause greater injury for tissues (Heusch 2013). Accumulated evidence has indicated that preconditioning with sevoflurane could attenuate the I/R injury both in vitro and in vivo (Guerrero-Orriach et al. 2017; Kunst \& Klein 2015; Obal et al. 2005), and there are multiple mechanisms involved in the cardioprotective effect of sevoflurane preconditioning (SPC), such as activation of KATP channel, to improve ion transport in the myocardial cell membrane, mitochondrial membrane and relieve $\mathrm{Ca}^{2+}$ overload (Zhang et al. 2012), activation of Adenosine A1 receptor as to reduce the production of reactive oxygen species (Lucchinetti et al. 2018), downregulation of apoptosis (Wei et al. 2017). However, the potential molecular mechanisms of SPC in myocardial protection still have not been fully elucidated.

Hypoxia-inducible factor-1 (HIF-1) is a protein that regulates the expression of an arrangement of hypoxiasensitive proteins beneath hypoxic conditions to preserve the survival of tissues (Semenza 2011). Considers have affirmed that HIF-1 is central to cardio-protection against I/R injury (Eckle et al. 2008). Pyruvate dehydrogenase kinase (PDK) is a key protein downstream of HIF-1 regulating energy metabolism. Now four isozymes of PDK1, PDK-2, PDK-3, and PDK-4 have been identified in PDK, they are mainly located in the mitochondrial matrix, in which PDK-1 is mainly expressed in the heart (Sugden \& Holness 2003). When cells are in a state of hypoxia, HIF-1 regulates the expression of downstream target protein PDK-1, which changes the energy metabolism of cardiomyocytes from the tricarboxylic acid cycle to glycolysis (Holness \& Sugden 2003). Although glycolysis is an inefficient way of energy production compared with mitochondrial aerobic oxidation, it plays an important role in sustaining basic life activities under the conditions of ischemia/hypoxia. Thus, we investigated whether the myocardial protective effect of SPC was associated with the regulation of glycolysis activity by activating the HIF-1a/PDK-1 signaling pathway in $\mathrm{H} 9 \mathrm{c} 2$ cardiomyocytes.

In the present study, H9c2 cardiomyocytes were used to establish a hypoxia/reoxygenation (H/R) induced myocardial cell injury model. To explore the molecular mechanism of SPC against H/R injury of cardiomyocytes 
by studying the activities of key enzymes and energy metabolism of glycolysis.

61

\section{Materials and methods}

\section{Reagent and antibodies}

TRYPSIN 0.25\% (1X) solution with EDTA, penicillin-streptomycin solution and DMEM/low glucose medium, phosphate-buffered saline (PBS) were purchased from Hyclone (Cat\#SH30042.01, SV30010, SH30021.01, SH30256.01; Hyclone, US). Fetal Bovine Serum (Cat\#10091148, GIBCO, New York, US). Dimethyl sulfoxide, Tris base, Glycine were purchased from Sigma (Cat\# V900090, V900483, V900144; Sigma, US).

Annexin V-PE apoptosis detection kit was purchased from Becton, Dickinson, and Company (Cat\#559763, US). Mitochondrial membrane potential detection kit (JC-1), 10仓TBST buffer, non-fat milk powder, sodium dodecyl sulfate, SDS-page gel preparation kit, $4 \diamond$ protein sample buffer (containing $\beta$-mercaptoethanol) were purchased from Solarbio (Cat\#M8650, T1081, S8010-100, P1200-2, P1016, Solarbio, Beijing, China). ATP detection kit and BCA protein quantitative kit were purchased from Beyotime (Cat\#s0026, P0012, Beyotime, Shanghai, China). The lactate dehydrogenase (LDH) kit was purchased from Nanjing Jiancheng Biotech Co. Ltd (Cat\# A020-2).

Rabbit-anti-HIF-1 alpha monoclonal antibody [EPR16897] (ab179483), Rabbit-anti-GAPDH antibody [EPR16891] (ab181602-100), Rabbit-anti-Bcl-2 monoclonal antibody (ab62557), Rabbit-anti-Bax monoclonal antibody (ab32503), horseradish peroxidase (HRP)-conjugated goat anti-rabbit immunoglobulin G (IgG) (ab205718), 10^RIPA lysate (ab156034), Protease Inhibitor Cocktail, (ab201111), ECL Substrate Kit (High Sensitivity) (ab133406) were purchased from Abcam Cambridge, MA, US. Rainbow protein pre-staining marker, 10 to $180 \mathrm{kDa}$ (26616, thermo-fermentas, MA, US). Rabbit-anti-PDK-1 (Cat\# BF0312, Affinity Biosciences, Changzhou, China). Lificiguat (YC-1) was purchased from Selleck (Cat\#S7958,Shanghai, China).

\section{Cell culture and processing}

The H9c2 rat embryonic cardiomyocyte cell line was obtained from Procell Life Science \& Technology Co., Ltd., China. The cell culture conditions consisted of DMEM (low sugar, 5mM) medium+10\% (v/v) FBS (Gibco, USA) $+1 \%$ (v/v) Penicillin/Streptomycin solution (Gibco, USA) at $37^{\circ} \mathrm{C}, 5 \% \mathrm{CO} 2$, and saturated humidity. The $\mathrm{H} 9 \mathrm{c} 2$ cells were passaged at the ratio of 1:4 when the cell confluence reached $90 \%$ (by visual estimate) in $55 \mathrm{~cm}^{2}$ culture plate. Cells were cultured for $48 \mathrm{~h}$ at $37^{\circ} \mathrm{C}$ in a $5 \% \mathrm{CO} 2$ incubator. The supernatant was discarded when the cells grew to $90 \%$ confluency, After gently washing the adherent cells with PBS twice, the serum-free DMEM (low sugar, 5mM) medium was added. Treatment with Lificiguat (YC-1, 10 $\mu \mathrm{M})$ was added before sevoflurane preconditioning. Plates with H9c2 cardiomyocytes were then placed in a sealed chamber (Modular 
91 Incubator Chamber, MIC1; Billups-Rothenberg, Inc., Del Mar, CA, USA; http://www.brincubator.com/) filled

92 with $95 \% \mathrm{~N}_{2}$ and $5 \% \mathrm{CO}_{2}$ to achieve an oxygen-deficient environment. Ventilation at $5 \mathrm{~L} / \mathrm{min}$ for $15 \mathrm{~min}$ was

93 used to achieve a 1\% lower oxygen concentration in the chamber (The oxygen indicator card was used to reflect

94 the oxygen concentration. Its color will change from blue to red when the oxygen concentration is less than $0.1 \%$

95 in the sealed chamber). Cells were incubated at $37^{\circ} \mathrm{C}$ for $3 \mathrm{~h}$, and the PBS was removed and replaced with fresh

96 medium containing 10\% FBS. Longer $3 \mathrm{~h}$ incubation in $95 \%$ air and $5 \% \mathrm{CO} 2$ at $37^{\circ} \mathrm{C}$ was performed as

97 reoxygenation. The plates of the CON group were kept in normoxic conditions for the corresponding times.

98 Experimental protocol

99 The H9c2 cardiomyocytes were randomly divided into four groups(Fig.1).

100 (1) Control (CON) group: H9c2 cells were continuously cultured in DMEM low-glucose medium (5mM) 101 containing 10\% FBS without any interventions but with inhalation of pure oxygen (100\% oxygen) for 15 min at 102 the same time interval when the SPC group was exposed to sevoflurane.

103 (2) Hypoxia/reoxygenation (H/R) group: H9c2 cells were cultured in low glucose concentration medium for 48 $104 \mathrm{~h}$, removed, washed with PBS two times and inhaled pure oxygen (100\% oxygen) for 15 min and placed in an 105 airtight container with 95\% N2 and 5\% CO2 for $3 \mathrm{~h}$, then reoxygenation (95\% air and 5\% CO2) with the addition 106 of fresh low glucose DMEM with $10 \%$ FBS at $37^{\circ} \mathrm{C}$, for a total $3 \mathrm{~h}$ of reoxygenation.

107 (3) Sevoflurane preconditioning group (SPC): H9c2 cells were exposed to 2.4\% sevoflurane for 15 min before 108 hypoxia after incubation in the medium for $48 \mathrm{~h}$. the later steps are all same to the H/R group.

109 (4) Hypoxia-inducible factor-1a inhibitor YC-1 group (S+Y): Lificiguat (YC-1) was added before sevoflurane 110 preconditioning, after treatment with sevoflurane preconditioning, the later steps are all same to the SPC group.

111 Measurement of lactate dehydrogenase(LDH)

112 Data were collected as described previously (Yu et al. 2016). Cell supernatant (0.1ml) was collected from each 113 group's plate $1 \mathrm{~min}$ after reoxygenation. The absorbance values were measured at $450 \mathrm{~nm}$, and then put the 114 absorbance values into the formula $(\mathrm{LDH}(\mathrm{U} / \mathrm{L})=[($ Determination OD-Control OD) $($ Standard OD-Blank $115 \mathrm{OD})] \times 0.2 \mu \mathrm{mol} / \mathrm{ml} \times 1,000), \mathrm{LDH}$ activity was expressed as international units per liter (IU/L).

\section{Measurement of cell viability}

118 Data were collected as described previously (Yang et al. 2019). Each experiment was repeated three times, and 119 each group was repeated twice. 
121

122

123

124

125

126

127

128

129

130

131

132

133

134

135

136

137

138

139

140

141

142

143

144

145

146

147

148

149

150

\section{Flow cytometry assay}

The percentage of cells apoptosis was measured using the PE Annexin-V Apoptosis Detection Kit I (BD Biosciences Pharmingen, US). In brief, cells were collected and stained with annexin-V and 7-AAD as per the manufacturer's instrument. The apoptotic cells were identified by flow cytometry (Beckman Coulter, US).

\section{Detection of key enzymes in glycolysis}

The content of the key enzymes of glycolysis was detected by the Elisa High-end detection kits (Jianglai Biotech Co. Ltd, China). The key enzymes of glycolysis, including that of Hexokinase (HK), 6-phosphofructokinase-1 (PFK-1), Pyruvate kinase (PK), were measured. Wash the cells twice with pre-cooled PBS. RIPA lysis buffer was used to lyse the cells on ice for $30 \mathrm{~min}$, then the cell lysate was collected by EP tube. Centrifugation: 16,000 $\mathrm{rpm}, 4^{\circ} \mathrm{C}, 10 \mathrm{~min}$, collecting the supernatant, and waiting for the test. The standard curve was made on enzyme coated plate with $50 \mu \mathrm{l}$ different concentrations of a standard substance. $40 \mu \mathrm{l}$ of sample diluent was added to the enzyme coated plate, and then $10 \mu \mathrm{l}$ of the sample to be tested was added. $100 \mu \mathrm{l}$ of enzyme-labeled reagent was added into each well. The mixture was incubated at $37^{\circ} \mathrm{C}$ for $60 \mathrm{~min}$, then washed 5 times and left to dry. Chromogenic agent solutions A and B $(50 \mu \mathrm{leach})$ were added and incubated at room temperature for $15 \mathrm{~min}$ in the dark. After adding $50 \mu \mathrm{l}$ of the termination solution, the OD values of each pore were measured by an Enzyme labeling instrument at $450 \mathrm{~nm}$.

\section{Detection of glycolysis energy}

The level of intracellular ATP was detected using the ATP Bioluminescence Assay Kit (Beyotime, Shanghai, China). The cells lysate was added, fully lysed, and centrifuged at $15,000 \mathrm{rpm}$ for $10 \mathrm{~min}$ at $4^{\circ} \mathrm{C}$. The supernatant was collected into an EP tube, waiting for the test. Take $200 \mu \mathrm{l}$ ATP detection reagent and add $1800 \mu 1$ ATP detection reagent diluent to prepare a $2 \mathrm{ml}$ ATP detection working solution. The ATP standard solution was diluted into an appropriate concentration gradient with ATP detection lysate and made a standard curve using concentrations of $0.01,0.03,0.1,0.3,1,3$ and $10 \mu \mathrm{M}$. After adding $100 \mu \mathrm{l}$ ATP detection working solution and $20 \mu 1$ sample into the detection well, and then measured with multifunctional enzyme marker after two seconds interval.

\section{Detection of mitochondrial membrane potential}

JC-1 (Millipore, USA) is used to assess the redistribution of mitochondrial membrane potential $(\Delta \Psi \mathrm{m})$. 
151 According to the manufacturer's instruction, the H9c2 cells were incubated with $10 \mathrm{nmol} / \mathrm{L}$ JC-1 staining 152 solution at $37^{\circ} \mathrm{C}$ free light for $10 \mathrm{~min}$. The images were captured using a fluorescence inverted microscope 153 (LEICA-DMI4000B, Germany). Thirty randomly chosen cardiomyocytes per treatment group were analyzed 154 ( $\mathrm{n}=3$ independent experiments with 10 incubated cardiomyocytes per experiment). Red and green fluorescence 155 intensities were analyzed respectively using Image J software as described (Sun et al. 2019), and the ratio of the $156 \mathrm{red} / \mathrm{green}$ fluorescence was proportional to the $\Delta \Psi \mathrm{m}$.

157

158

159

160

161

162

163

164

165

166

167

168

169

170

171

172

173

174

175

176

177

178

179

180

181

\section{Western blot analysis}

The HIF-1a, PDK-1, Bax, Bcl-2, and glyceraldehyde 3-phosphate dehydrogenase (GAPDH) proteins were detected with the antibodies rabbit monoclonal anti-HIF-1a, rabbit monoclonal anti-GAPDH, rabbit monoclonal anti-Bax, rabbit monoclonal anti-Bcl-2 (Abcam, US), rabbit monoclonal anti-PDK-1 (Affinity, USA), Proteins were extracted with $100 \mu$ Lysis Solution (Radioimmunoprecipitation assay buffer/Phenylmethanesulfonyl fluoride $(\mathrm{PMSF})=100: 1)$ for each plate. The protein concentrations were measured using a BCA kit (Solarbio, Beijing, China) and all the concentrations were adjusted to the lowest protein concentration with the RIPA lysate. The supernatant was mixed with $5 \diamond$ loading buffer and heated for $5 \mathrm{~min}$ at $100{ }^{\circ} \mathrm{C}$, and then 30 micrograms of the sample were subjected to electrophoresis using an SDS-PAGE gel system, transferred to Polyvinylidene fluoride (PVDF) Western blotting membranes (Roche, Germany) and blocked in 5\% non-fat milk at $37^{\circ} \mathrm{C}$ for 2 h. Diluted primary antibodies to HIF-1a [EPR16897] (1:1000, Abcam, ab179483), Bax (1:500, Abcam, ab32503), GAPDH (1:1000, Abcam, ab181602-100), PDK-1 (1:1000, Affinity Biosciences Cat\#BF0312) and Bcl-2 (1:500, Abcam, ab62557) were added, and the membrane was incubated overnight $\left(4^{\circ} \mathrm{C}\right)$. The membrane was washed with TBST solution for 3 times and incubated with HRP-conjugated secondary antibody (1:10,000, Abcam, ab205718) for $2 \mathrm{~h}$ at room temperature. High Sensitivity ECL Substrate Kit (Abcam, ab133406) was used for visualization and imaging. Signals were detected and quantified with Image Lab 4.0 software (Bio-Rad Laboratories, US).

\section{Statistical analysis}

SPSS 20.0 statistical software was used for the data analysis. All values were expressed as the mean \pm standard deviation. The within-group comparisons were performed using the analysis of variance of repeated measurement design. Pairwise comparison in multiple groups was conducted with SNK method. $P<0.05$ was considered significant. GraphPad Prism 5.0 was used to prepare graphs. 


\section{RESULTS}

SPC reduced LDH level, cell death and expression of protein Bax, increased cell viability and expression of protein Bcl-2 following simulated Hypoxia/Reoxygenation (H/R)

To observe the effect of SPC on H/R induced cell injury, the H9c2 cells were subjected to LDH level, cell viability, flow cytometry, and western blot analysis. In this study, The cell viability of the H/R group was significantly lower than the CON group $(P<0.05)$, while the cell viability of the SPC group was increased compared to the H/R group ( $P<0.05$, Fig.2A). The LDH assay showed that compared to the H/R group, the LDH activity of the SPC group was decreased $(P<0.05)$, while the LDH activity did not significantly differ between the $\mathrm{H} / \mathrm{R}$ and $\mathrm{S}+\mathrm{Y}$ groups $(P>0.05$, Fig.2B). Compared to the CON group, the cells apoptotic rate of $\mathrm{H} / \mathrm{R}$ and $\mathrm{S}+\mathrm{Y}$ groups were significantly increased $(P<0.05)$, while the cells apoptotic rate of the SPC group was decreased compared to the $\mathrm{H} / \mathrm{R}$ group $(P<0.05$, Fig. $2 \mathrm{C})$. Flow cytometry to measure apoptosis distribution graphs were shown in Fig.D-G. The expression levels of apoptosis-related proteins were measured by using western blot analysis. Western blot images of Bcl-2 protein and Bax protein were shown in Fig.H-I. Compared to the CON group, the ratio of Bcl-2/GAPDH of $\mathrm{H} / \mathrm{R}$ and $\mathrm{S}+\mathrm{Y}$ groups did not have a significant difference $(P>0.05)$, however, the ratio of Bcl-2/GAPDH of SPC group was significantly increase ( $P<0.05$, Fig.2J). Compared to the $\mathrm{CON}$ group, the ratio of $\mathrm{Bax} / \mathrm{GAPDH}$ of $\mathrm{H} / \mathrm{R}$ and $\mathrm{S}+\mathrm{Y}$ groups were significantly increased, but the ratio of Bax/GAPDH of SPC was decreased $(P<0.05$, Fig.2K).

\section{Sevoflurane preconditioning increases the activity of key enzymes and ATP production in glycolysis}

The HK, PFK-1, and PK activities at the end of equilibration did not significantly differ among groups ( $P>0.05)$. The HK, PFK-1, and PK activities at the end of reoxygenation were significantly increased in all groups compared to the activities at the end of equilibration $(P<0.05)$. The comparison of all groups at the end of reoxygenation showed that the HK, PFK-1, and PK activities in the SPC group were significantly increased compared to those in the $\mathrm{H} / \mathrm{R}$ group $(P<0.05$, Fig. 3A, 3B and 3C).

The concentration of ATP at the end of equilibration did not significantly differ among groups $(P>0.05)$. The ATP assay showed that compared with the Con group, the ATP concentration of $\mathrm{H} / \mathrm{R}, \mathrm{SPC}$ and $\mathrm{S}+\mathrm{Y}$ groups were significantly decreased $(P<0.05)$. Compared to the H/R group, the ATP concentration of the SPC group was increased, while the $\mathrm{S}+\mathrm{Y}$ group was decreased $(P<0.05$, Fig. 3D).

\section{Protective effects of SPC on H/R injury of $\mathrm{H} 9 \mathrm{c} 2$ cells}


213

$\Delta \Psi \mathrm{m}$, a sign of early-stage apoptosis (Marchetti et al. 1996) was evaluated in H9c2 using JC-1 staining. Representative images of JC-1 in H9c2 cells were shown in Fig.4A. The result showed that the ratio of red to green fluorescence intensity in the SPC group was higher than the $\mathrm{H} / \mathrm{R}$ group $(P<0.05)$. However, After the application of hypoxia-inducible factor- $1 \alpha$ inhibitor YC-1, there was no significant difference in the ratio of red to green fluorescence intensity between $\mathrm{H} / \mathrm{R}$ group and $\mathrm{S}+\mathrm{Y}$ group $(P<0.05$, Fig. 4B)

\section{SPC increases the levels of HIF-1a and PDK-1 in the simulation of H/R injury}

At the end of reoxygenation, the expression of HIF-1a of SPC and H/R groups were all increased compared to the Con group $(P<0.05)$. Western blot images of HIF-1a protein and PDK-1 protein were shown in Fig.A-B. Compared to the H/R group, the expression of HIF-1a of the SPC group was increased, while the S+Y group was decreased $(P<0.05$, Fig. 5C). Compared to the CON group, the expression of PDK-1 of SPC and H/R groups were all increased $(P<0.05)$, while the $\mathrm{S}+\mathrm{Y}$ group did not have statistical significance $(P>0.05)$. Compared to the SPC group, the expression of PDK-1 of the S+Y group was decreased $(P<0.05)$ when the inhibitor YC-1 was added into the culture medium (Fig. 5D).

\section{Discussion}

The results of our study confirmed that sevoflurane preconditioning (SPC) can effectively attenuate the Hypoxia/Reoxygenation $(\mathrm{H} / \mathrm{R})$ injury in $\mathrm{H} 9 \mathrm{c} 2$ cardiomyocytes. This finding is in line with the proven SPC cardiac protective effects reported in a related study (Wenlan et al. 2018). It is suggested that the cardioprotective effect of SPC may be related to the activation of the HIF-1a/PDk-1 pathway.

Sevoflurane is widely used in all types of clinical cardiac surgery as a representative inhalation anesthetic drug and is considered by the majority of scholars to be a landmark inhalation anesthesia drug. Some studies have found that the protective effect of sevoflurane on ischemic myocardium, such as sevoflurane preconditioning (SPC) or sevoflurane postconditioning (SPostC), is closely related to its mode of administration (De Hert et al. 2004; Qian et al. 2018; Wu et al. 2017). Ischemic preconditioning (IPC) is the most effective myocardial protection strategy found so far (Cai et al. 2003), and SPC can produce myocardial protective effects similar to IPC (Qian et al. 2018) and has stronger clinical maneuverability. In the study, H9c2 cardiomyocytes were used as the research object to simulate the H/R model to explore the molecular mechanism of myocardial protection of SPC, to provide a theoretical basis for scientific and clinic research.

A member of the hypoxia-inducible factor family (HIFs) is HIF-1. Due to the inhibition of the activity of prolyl-4-hydroxylase domain-containing enzymes (BHD) under anoxia situations, the ubiquitination of HIF-1 is 
244

245

246

247

248

249

250

251

252

253

254

255

256

257

258

259

260

261

262

263

264

265

266

267

268

269

270

271

272

273

274

275

276

277

278

reduced. In the nucleus, it binds to the target gene promoter's hypoxia response element and regulates the transcription of a series of downstream target genes which contribute to cell survival under hypoxic conditions (Prabhakar \& Semenza 2015). PDK-1 is a switch protein that regulates energy metabolism downstream of HIF-1 (Semenza 2011). Glucose will form pyruvate under aerobic conditions and then enter into the tricarboxylic acid cycle to be oxidized to carbon dioxide and water, while in the anaerobic condition, pyruvate undergoes glycolysis under the action of PDK-1 to produce lactic acid (Akram 2013). In this study, SPC and HIF-1a inhibitor YC-1 were used as intervention methods for grouping study. Western Blot protein detection technique was used to quantify the contents of target protein HIF-1a and PDK-1. The results showed that the contents of HIF-1a and PDK-1 were significantly increased in the SPC group, while the contents were significantly decreased after using the inhibitor of HIF-1a. It is suggested that the myocardial protective effect of SPC may be related to activation of the HIF-1a/PDK-1 signal pathway.

ATP is the direct energy for all kinds of life activities. The myocardium has a high energy demand, but there is basically no energy reserve (Lopaschuk et al. 2010). Therefore, the heart must continuously produce a large amount of ATP to maintain muscle contraction and ion homeostasis. Most of the ATP production (about 9095\%) comes from oxidative phosphorylation of mitochondria and the rest from glycolysis. Mitochondria can use a variety of energy substrates to produce ATP, including fatty acids, carbohydrates, ketones and amino acids, among which fatty acid oxidation is the main source of ATP in the heart (Itoi \& Lopaschuk 1993). What's more, normal myocardium has a lot of "metabolic flexibility" (Kolwicz et al. 2013; Ritterhoff \& Tian 2017), allowing it to switch back and forth between fatty acid and carbohydrate oxidation, depending on the load of the heart, the energy substrate provided to the heart, and the hormonal and nutritional status (Taegtmeyer et al. 2004). The contribution for myocardial ATP production by glycolysis was increased under the condition of myocardial ischemia/hypoxia (Akram 2013). Glycolysis is a dynamic process, which provides ATP for the body in the early stage of hypoxia. However, with the extension of time, glycolysis will produce excessive acid metabolites, which will lead to acidosis and aggravate the apoptosis and damage of the body (Dang et al. 2005). The study confirmed that the glycolytic pathway content of key rate-limiting enzymes (HK, PFK-1, and PK) in cardiomyocytes may indirectly reflect the state of energy metabolism of cardiomyocytes (Volker et al. 1995). And after hypoxia injury, the levels of HK, PFK-1, and PK increased and the glycolytic activity of cardiomyocytes was significantly increased in early hypoxia (Guo et al. 2017; Teng et al. 2010) and cardiomyocyte glycolysis activity reached its peak at 1-3 hours of hypoxia, Since then, and has been decreasing (Dang et al. 2005). In our experiment, the time limit of cell hypoxia damage was set as 3 hours and the results showed that activity of the key enzymes of glycolysis of H/R, SPC and Y+S groups were significantly increased compared to CON group, and the activity of the key glycolytic enzymes was the highest in the SPC group. The ATP concentration of SPC was also higher than the H/R group. There are evidences that HIF-1a is also the main regulator of glycolytic enzyme expression and the key transcription factor that drives glycolysis under anoxic conditions (Semenza 2010; Xie et al. 2015). These evidences led us to hypothesize that SPC could optimize the glycolysis in early hypoxia by activating the 
HIF-1a/PDK-1 signal pathway.

Mitochondria are not only the main place of energy metabolism in eukaryotes but also the main target of ischemic/hypoxic injury (Halestrap \& Richardson 2015). The level of mitochondrial membrane potential is an index of early cardiomyocyte apoptosis (Marchetti et al. 1996). In this study, JC-1 was used for cell staining, and the levels of mitochondrial membrane potential in different experimental groups were compared and the apoptosis-related proteins Bcl-2 and Bax were detected by WB. The results showed that the levels of antiapoptotic protein $\mathrm{Bcl}-2$ and mitochondrial membrane potential in the SPC group were higher than those in $\mathrm{H} / \mathrm{R}$ and $\mathrm{Y}+\mathrm{S}$ groups, indicating that SPC could stabilize cardiomyocyte mitochondrial membrane potential and reduce the rate of apoptosis induced by $\mathrm{H} / \mathrm{R}$ injury in $\mathrm{H} 9 \mathrm{c} 2$ cardiomyocytes.

\section{Limitations}

Several limitations of this study should be noted. Firstly, we only tested the hypothesis of the cardioprotective effect of SPC at the cardiomyocyte level, and it should be further verified at the animal level in the future. Secondly, the concentration of SPC used in this study was $2.4 \%$, and the effect of other concentrations on the results should also be investigated. Thirdly, we only observed the potential HIF-1a/PDK-1 signaling pathways mechanisms in the myocardial protective function of SPC, further investigation is needed to identify essential components in these complex signal transduction cascades that mediate SPC.

\section{Conclusions}

In summary, this study demonstrated that the cardioprotective effect of SPC are associated with the activation of the HIF-1a/PDk-1 pathway. The mechanism may be related to an increase in the expression of HIF-1a, PDK-1 and Bcl-2 after SPC, which leads to increasing the contents of key enzymes and ATP concentration of glycolysis, thereby stabling mitochondrial membrane potential and reducing apoptosis.

\section{Acknowledgments}

This work was supported by the National Natural Science Foundation (Grant No. U1603129) and the National Natural Science Funds (Grant No. 81660522) of China. The funders had no role in study design, data collection and analysis, decision to publish, or preparation of the manuscript. 


\section{Competing Interests}

309 The authors declare that they have no competing interests.

\section{Author Contributions}

312 Tianliang Hou conceived and designed the experiments, performed the experiments, analyzed the data, 313 contributed reagents/materials/analysis tools, prepared figures and/or tables, approved the final draft.

314 Haixia Wang, Haiping Ma conceived and designed the experiments, performed the experiments.

315 Chunling Chen performed the experiments, contributed reagents/materials/analysis tools.

316 Ahmed Mohamed Ahmed modify grammar and English language usage of this article

317 Jianrong Ye analyzed the data, prepared figures and/or tables, authored or reviewed drafts of the paper.

318 Hong Zheng conceived and designed the experiments, wrote the paper and reviewed drafts of the paper.

\section{References}

Akram M. 2013. Mini-review on glycolysis and cancer. J Cancer Educ 28:454-457. 10.1007/s13187-013-0486-9

Cai Z, Manalo DJ, Wei G, Rodriguez ER, Fox-Talbot K, Lu H, Zweier JL, and Semenza GL. 2003. Hearts from rodents exposed to intermittent hypoxia or erythropoietin are protected against ischemia-reperfusion injury. Circulation 108:79-85. 10.1161/01.cir.0000078635.89229.8a

Dang YM, Huang Y, and Zhou JL. 2005. Effects of hypoxia inducible factor-1 $\alpha$ on glycolysis of rat cardiomyocytes under hypoxia Chinese Journal of burns 21:339-342.

De Hert SG, Van der Linden PJ, Cromheecke S, Meeus R, Nelis A, Van Reeth V, ten Broecke PW, De Blier IG, Stockman BA, and Rodrigus IE. 2004. Cardioprotective properties of sevoflurane in patients undergoing coronary surgery with cardiopulmonary bypass are related to the modalities of its administration. Anesthesiology 101:299-310. 10.1097/00000542-200408000-00009 DI, Kurz A, Szczeklik W, Berwanger O, Villar JC, Malaga G, Garg AX, Chow CK, Ackland G, Patel A, Borges FK, 
335

336

337

338

339

340

341

342

343

Thabane L, Rodseth RN, Buse GAL, Bhandari M, Garutti I, Jacka MJ, Schünemann HJ, Cortes OL, Coriat P, Dvirnik N, Botto F, Pettit S, Jaffe AS, and Guyatt GH. 2017. Association of Postoperative High-Sensitivity Troponin Levels With Myocardial Injury and 30-Day Mortality Among Patients Undergoing Noncardiac Surgery. Jama 317:1642-1651. $10.1001 /$ jama.2017.4360

Eckle T, Köhler D, Lehmann R, El Kasmi K, and Eltzschig HK. 2008. Hypoxia-inducible factor-1 is central to cardioprotection: a new paradigm for ischemic preconditioning. Circulation 118:166-175. 10.1161/circulationaha.107.758516

Guerrero-Orriach JL, Escalona Belmonte JJ, Ramirez Fernandez A, Ramirez Aliaga M, Rubio Navarro M, and Cruz Mañas J. 2017. Cardioprotection with halogenated gases: how does it occur? Drug Des Devel Ther 11:837-849. 10.2147/dddt.s127916

Guo H, Zheng H, Wu J, Ma HP, Yu J, and Yiliyaer M. 2017. The key role of microtubules in hypoxia preconditioning-induced nuclear translocation of HIF-1 $\alpha$ in rat cardiomyocytes. PeerJ 5:e3662. 10.7717/peerj.3662

Halestrap AP, and Richardson AP. 2015. The mitochondrial permeability transition: a current perspective on its identity and role in ischaemia/reperfusion injury. J Mol Cell Cardiol 78:129-141. 10.1016/j.yjmcc.2014.08.018

Heusch G. 2013. Cardioprotection: chances and challenges of its translation to the clinic. Lancet 381:166-175. 10.1016/s01406736(12)60916-7

Holness MJ, and Sugden MC. 2003. Regulation of pyruvate dehydrogenase complex activity by reversible phosphorylation. Biochem Soc Trans 31:1143-1151. 10.1042/bst0311143

Itoi T, and Lopaschuk GD. 1993. The contribution of glycolysis, glucose oxidation, lactate oxidation, and fatty acid oxidation to ATP production in isolated biventricular working hearts from 2-week-old rabbits. Pediatr Res 34:735-741. $10.1203 / 00006450-199312000-00008$

Kolwicz SC, Jr., Purohit S, and Tian R. 2013. Cardiac metabolism and its interactions with contraction, growth, and survival of cardiomyocytes. Circ Res 113:603-616. 10.1161/circresaha.113.302095

Kunst G, and Klein AA. 2015. Peri-operative anaesthetic myocardial preconditioning and protection - cellular mechanisms and clinical relevance in cardiac anaesthesia. Anaesthesia 70:467-482. 10.1111/anae.12975

Lopaschuk GD, Ussher JR, Folmes CD, Jaswal JS, and Stanley WC. 2010. Myocardial fatty acid metabolism in health and disease. Physiol Rev 90:207-258. 10.1152/physrev.00015.2009

Lucchinetti E, Lou PH, Gandhi M, Clanachan AS, and Zaugg M. 2018. Differential Effects of Anesthetics and Opioid Receptor Activation on Cardioprotection Elicited by Reactive Oxygen Species-Mediated Postconditioning in Sprague-Dawley Rat Hearts. Anesth Analg 126:1739-1746. 10.1213/ane.0000000000002676

Marchetti P, Castedo M, Susin SA, Zamzami N, Hirsch T, Macho A, Haeffner A, Hirsch F, Geuskens M, and Kroemer G. 1996. 
364

365

366

367

368

369

370

371

372

373

374

375

376

377

378

379

380

381

382

383

384

Mitochondrial permeability transition is a central coordinating event of apoptosis. J Exp Med 184:1155-1160. $10.1084 /$ jem.184.3.1155

Obal D, Dettwiler S, Favoccia C, Scharbatke H, Preckel B, and Schlack W. 2005. The influence of mitochondrial KATP-channels in the cardioprotection of preconditioning and postconditioning by sevoflurane in the rat in vivo. Anesth Analg 101:12521260. 10.1213/01.ane.0000181336.96511.32

Prabhakar NR, and Semenza GL. 2015. Oxygen Sensing and Homeostasis. Physiology (Bethesda) 30:340-348. 10.1152/physiol.00022.2015

Qian B, Yang Y, Yao Y, Liao Y, and Lin Y. 2018. Upregulation of vascular endothelial growth factor receptor-1 contributes to sevoflurane preconditioning-mediated cardioprotection. Drug Des Devel Ther 12:769-776. 10.2147/dddt.s162577

Ritterhoff J, and Tian R. 2017. Metabolism in cardiomyopathy: every substrate matters. Cardiovasc Res 113:411-421. $10.1093 / \mathrm{cvr} / \mathrm{cvx} 017$

Semenza GL. 2010. HIF-1: upstream and downstream of cancer metabolism. Curr Opin Genet Dev 20:51-56. 10.1016/j.gde.2009.10.009

Semenza GL. 2011. Oxygen sensing, homeostasis, and disease. N Engl J Med 365:537-547. 10.1056/NEJMra1011165

Sugden MC, and Holness MJ. 2003. Recent advances in mechanisms regulating glucose oxidation at the level of the pyruvate dehydrogenase complex by PDKs. Am J Physiol Endocrinol Metab 284:E855-862. 10.1152/ajpendo.00526.2002

Sun MH, Chen XC, Han M, Yang YN, Gao XM, Ma X, Huang Y, Li XM, Gai MT, Liu F, Ma YT, and Chen BD. 2019. Cardioprotective effects of constitutively active MEK1 against $\mathrm{H}(2) \mathrm{O}(2)$-induced apoptosis and autophagy in cardiomyocytes via the ERK1/2 signaling pathway. Biochem Biophys Res Commun 512:125-130. 10.1016/j.bbrc.2019.03.008

Taegtmeyer H, Golfman L, Sharma S, Razeghi P, and van Arsdall M. 2004. Linking gene expression to function: metabolic flexibility in the normal and diseased heart. Ann N Y Acad Sci 1015:202-213. 10.1196/annals.1302.017

Teng M, Dang YM, Zhang JP, Zhang Q, Fang YD, Ren J, and Huang YS. 2010. Microtubular stability affects cardiomyocyte glycolysis by HIF-1alpha expression and endonuclear aggregation during early stages of hypoxia. Am J Physiol Heart Circ Physiol 298:H1919-1931. 10.1152/ajpheart.01039.2009

Volker KW, Reinitz CA, and Knull HR. 1995. Glycolytic enzymes and assembly of microtubule networks. Comp Biochem Physiol B Biochem Mol Biol 112:503-514. 10.1016/0305-0491(95)00096-8

Wei J, Chen S, Xue S, Zhu Q, Liu S, Cui L, Hua X, and Wang Y. 2017. Blockade of Inflammation and Apoptosis Pathways by siRNA Prolongs Cold Preservation Time and Protects Donor Hearts in a Porcine Model. Mol Ther Nucleic Acids 9:428- 
Wenlan L, Zhongyuan X, Shaoqing L, Liying Z, Bo Z, and Min L. 2018. MiR-34a-5p mediates sevoflurane preconditioning induced inhibition of hypoxia/reoxygenation injury through STX1A in cardiomyocytes. Biomed Pharmacother 102:153-159. 10.1016/j.biopha.2018.03.002

Wu J, Yu J, Xie P, Maimaitili Y, Wang J, Yang L, Ma H, Zhang X, Yang Y, and Zheng H. 2017. Sevoflurane postconditioning protects the myocardium against ischemia/reperfusion injury via activation of the JAK2-STAT3 pathway. PeerJ 5:e3196. 10.7717/peerj.3196

Xie N, Tan Z, Banerjee S, Cui H, Ge J, Liu RM, Bernard K, Thannickal VJ, and Liu G. 2015. Glycolytic Reprogramming in Myofibroblast Differentiation and Lung Fibrosis. Am J Respir Crit Care Med 192:1462-1474. 10.1164/rccm.201504$0780 \mathrm{OC}$

Yang L, Wu J, Xie P, Yu J, Li X, Wang J, and Zheng H. 2019. Sevoflurane postconditioning alleviates hypoxia-reoxygenation injury of cardiomyocytes by promoting mitochondrial autophagy through the HIF-1/BNIP3 signaling pathway. PeerJ 7:e7165. 10.7717/peerj.7165

Yu J, Wu J, Xie P, Maimaitili Y, Wang J, Xia Z, Gao F, Zhang X, and Zheng H. 2016. Sevoflurane postconditioning attenuates cardiomyocyte hypoxia/reoxygenation injury via restoring mitochondrial morphology. PeerJ 4:e2659. $10.7717 /$ peerj.2659

Zhang FJ, Ma LL, Wang WN, Qian LB, Yang MJ, Yu J, Chen G, Yu LN, and Yan M. 2012. Hypercholesterolemia abrogates sevoflurane-induced delayed preconditioning against myocardial infarct in rats by alteration of nitric oxide synthase signaling. Shock 37:485-491. 10.1097/SHK.0b013e318249b7b6 


\section{Figure 1}

A schematic representation of the experiment protocol

H9c2 cardiomyocytes were randomly divided into control (CON), hypoxia/reoxygenation $(\mathrm{H} / \mathrm{R})$, sevoflurane preconditioning $(\mathrm{SPC})$ and sevoflurane preconditioning $+\mathrm{HIF}-\mathrm{la}$ inhibitor $(S+Y)$ groups.

\begin{tabular}{|c|c|c|c|c|}
\hline \multirow[b]{2}{*}{$\mathrm{CON}$} & & $15 \mathrm{~min}$ & $\rightarrow 3 \mathrm{~h} \leftarrow$ & $\longrightarrow 3 \mathrm{~h} \longleftarrow$ \\
\hline & BASELINE & & \multicolumn{2}{|c|}{ NORMOXIA } \\
\hline H/R & BASELINE & & HYPOXIA & REOXYGENATION \\
\hline SPC & BASELINE & & HYPOXIA & REOXYGENATION \\
\hline $\mathbf{S}+\mathbf{Y}$ & BASELINE & & HYPOXIA & REOXYGENATION \\
\hline
\end{tabular}

T 1

Sevoflurane

YC-1+Sevoflurane 


\section{Figure 2}

SPC alleviated H9c2 cardiomyocytes hypoxia/reoxygenation injury

(A) Cell viability: Compared to the H/R group, the SPC group exhibited improved cell viability ( $n=6)$. (B) LDH activity: The LDH activity was reduced in the SPC group compared to the H/R group $(n=6)$. (C) Apoptosis rate: The apoptosis rate of the SPC group was lower than that of the H/R group. While the protective effects of SPC were inhibited after administration of YC-1 $(n=6)$. (D-G) Flow cytometry to measure apoptosis distribution graph. $(H)$ Western blot image of $\mathrm{BCl}-2$ protein. (I) Western blot image of Bax protein. (J) Bcl-2 protein expression. (K) Bax protein expression. Protein content was normalized to $\operatorname{GAPDH}(n=3 /$ group $)$. Data represent mean $\pm \mathrm{SD}\left({ }^{*} \mathrm{P}<0.05\right.$ vs $\mathrm{C}$ group, ${ }^{*} \mathrm{P}<0.05$ vs $\mathrm{H} / \mathrm{R}$ group, ${ }^{\circledR} \mathrm{P}<0.05$ vs $\mathrm{SPC}$ group) 

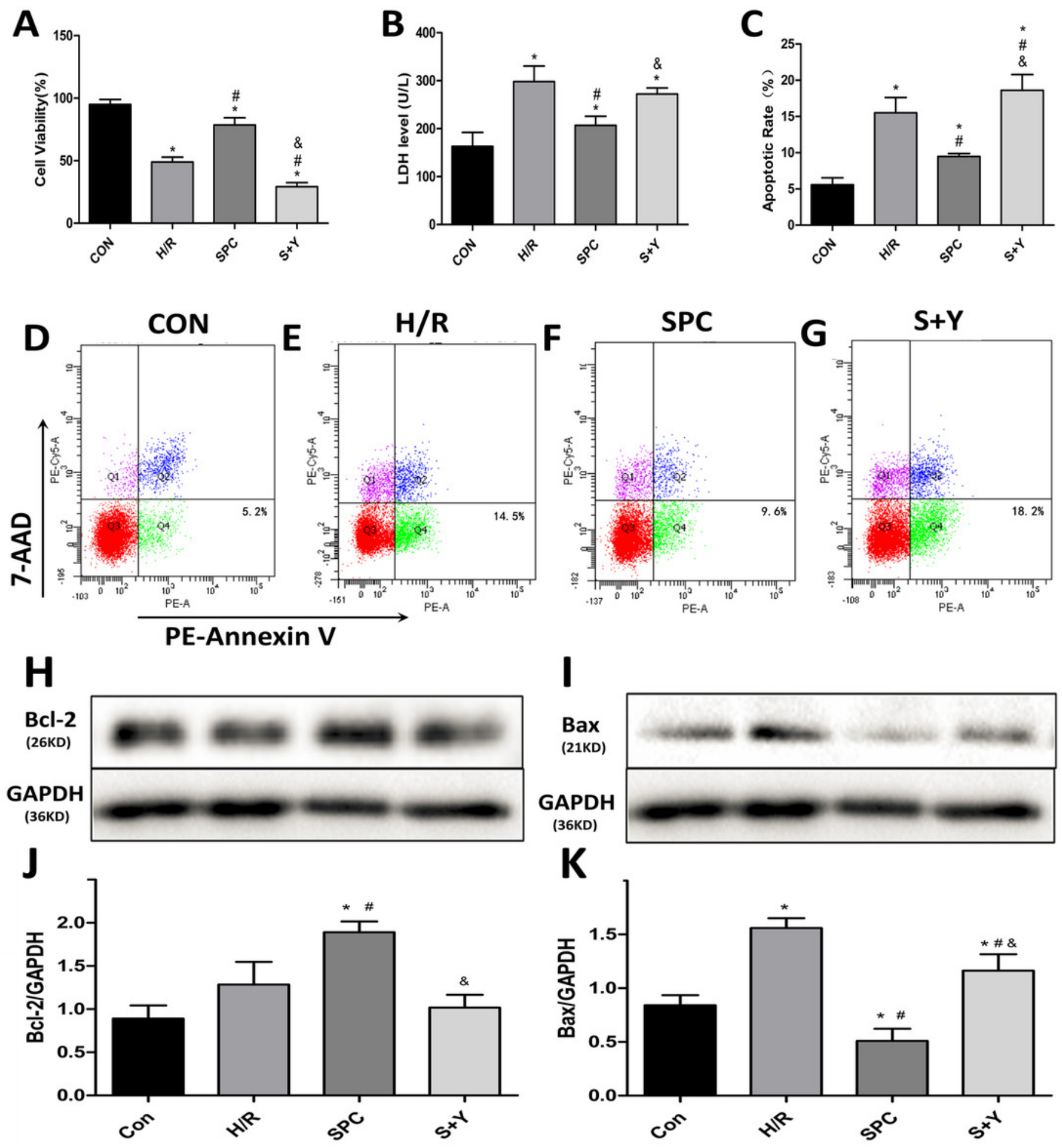
Figure 3

SPC improve glycolysis energy metabolism and key enzymes activity.

The end of equilibration (T1) and the end of reoxygenation (T2). (A)HK concentration.

(B)PFK-1 concentration. (C)PK concentration. (D)ATP concentration. Data are presented as the mean $\pm S D(n=6) .{ }^{*}<<0.05$ vs T1; T2 time point: ${ }^{\wedge} P<0.05$ vs $C O N, \# P<0.05$ vs $H / R$, $\& P<0.05$ vs SPC.
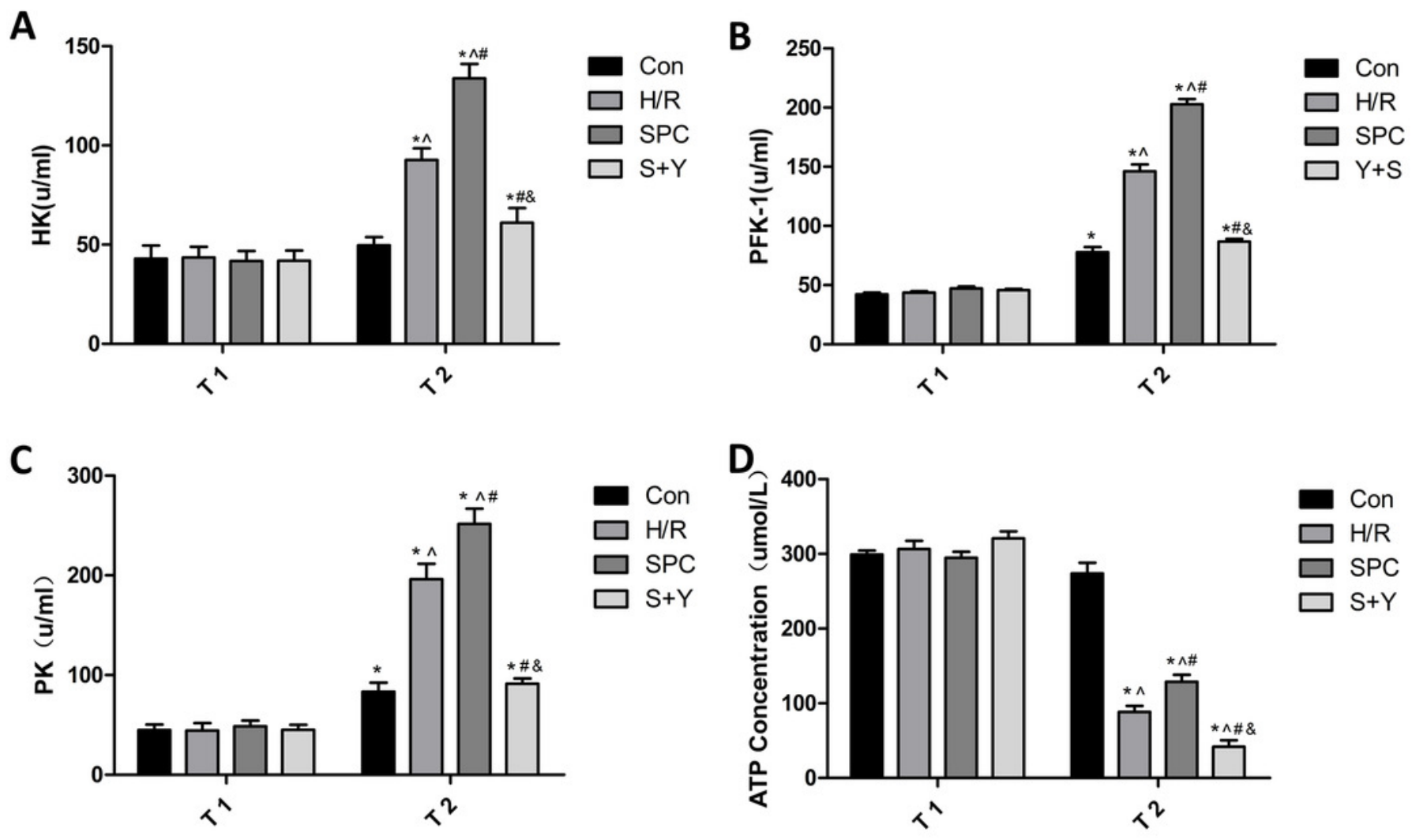


\section{Figure 4}

SPC stabilize the mitochondrial membrane potential $(\Delta \Psi \mathrm{m})$

(A) Representative images of JC-1 were capture using fluorescence inverted microscope in H9c2 cells. (B)The results showed that SPC increased the ratio of red to green fluorescence intensity. Data represent mean $\pm S D$ ( $n=3$ /group). ${ }^{*} P<0.05$ vs Con, ${ }^{\#} P<0.05 \mathrm{vs} H / R$, ${ }^{\&} P$ $<0.05$ vs SPC.

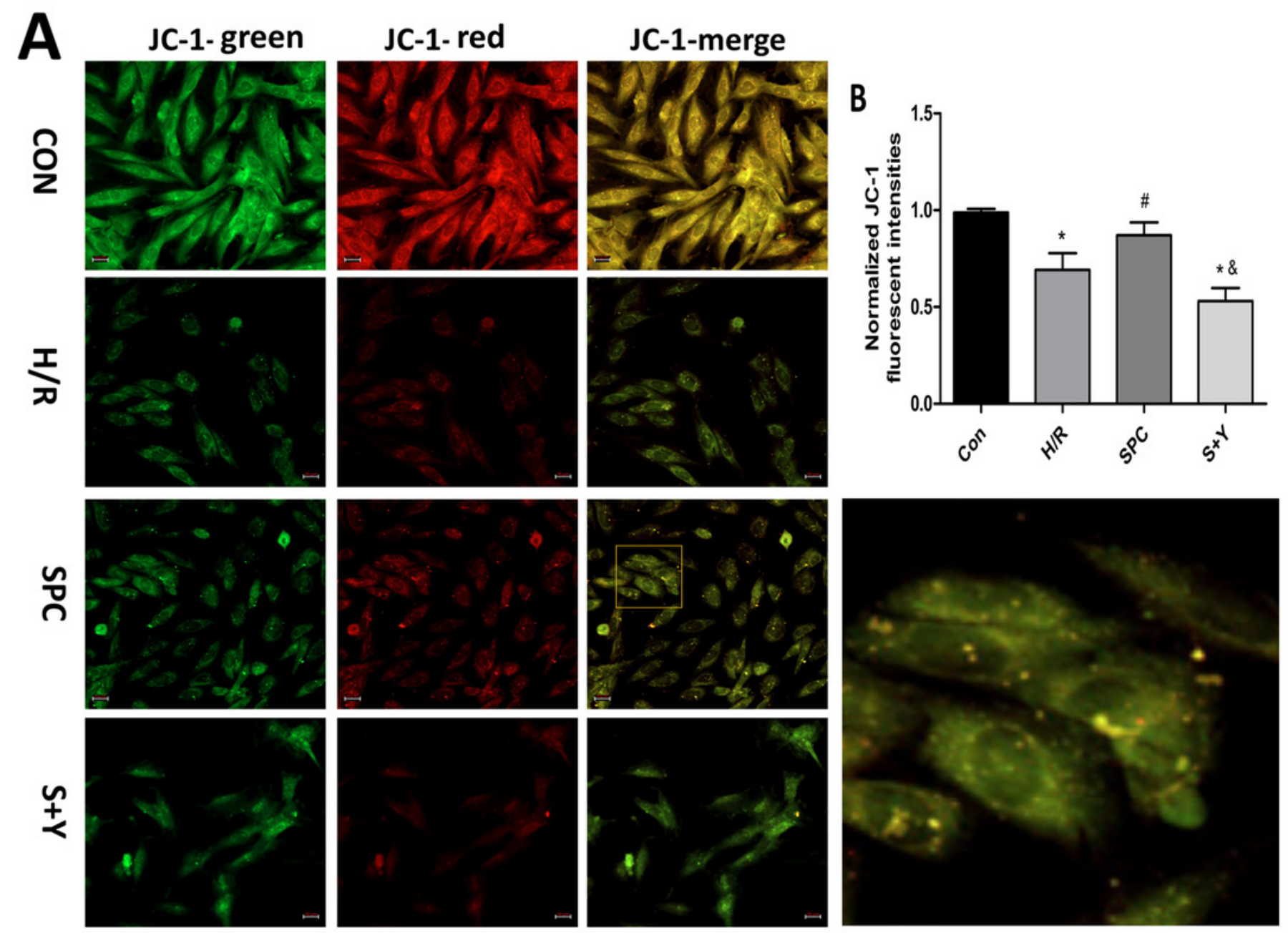


Figure 5

SPC upregulate the protein expression of HIF-1a and PDK-1.

(A) Western blot image of HIF-1a protein. (B) Western blot image of PDK-1 protein. (C)HIF-1a protein expression. (D)PDK-1 protein expression. Protein content was normalized to GAPDH. Data represent mean $\pm S D(n=3 /$ group). $* P<0.05$ vs Con, $\# P<0.05$ vs $H / R, \& P<0.05$ vs SPC.
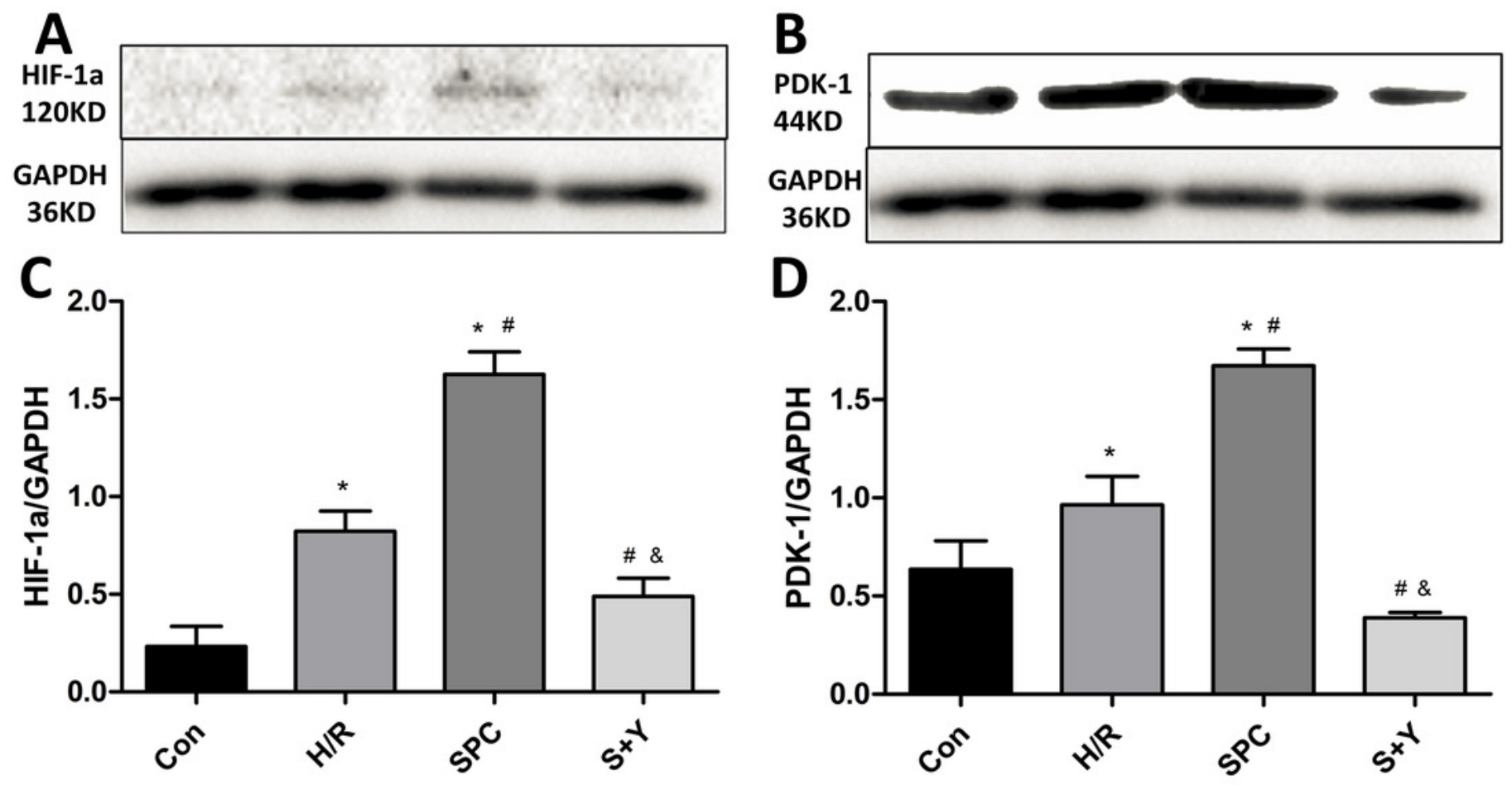